Keywords: clinical trial recruitment; informed consent; decision aids; randomised controlled trial; International Breast Cancer Intervention Study-II (IBIS-II); decisional conflict

\title{
Improving decision making about clinical trial participation - a randomised controlled trial of a decision aid for women considering participation in the IBIS-II breast cancer prevention trial
}

I Juraskova ${ }^{*}, 1$, P Butow ${ }^{1}$, C Bonner ${ }^{1}$, M L Bell ${ }^{1}$, A B Smith ${ }^{1}$, M Seccombe ${ }^{2}$, F Boyle ${ }^{1,2,3}$, L Reaby ${ }^{2}$, J Cuzick ${ }^{4}$ and J F Forbes ${ }^{2}$

${ }^{1}$ Centre for Medical Psychology and Evidence-based Decision-making (CeMPED), The University of Sydney, Sydney 2006, Australia; ${ }^{2}$ Australia and New Zealand Breast Cancer Trials Group, Australia University of Newcastle, Newcastle 2306, Australia; ${ }^{3}$ Pam McLean Centre, The University of Sydney, Sydney 2006, Australia and ${ }^{4}$ Cancer Research UK, Department of Epidemiology, Mathematics and Statistics, Wolfson Institute of Preventive Medicine, Queen Mary College, University of London, London E1 4NS, UK

Background: Decision aids may improve informed consent in clinical trial recruitment, but have not been evaluated in this context. This study investigated whether decision aids (DAs) can reduce decisional difficulties among women considering participation in the International Breast Cancer Intervention Study-II (IBIS-II) trial.

Methods: The IBIS-II trial investigated breast cancer prevention with anastrazole in two cohorts: women with increased risk (Prevention), and women treated for ductal carcinoma in situ (DCIS). Australia, New Zealand and United Kingdom participants were randomised to receive a DA (DA group) or standard trial consent materials (control group). Questionnaires were completed after deciding about participation in IBIS-II (post decision) and 3 months later (follow-up).

Results: Data from 112 Prevention and 34 DCIS participants were analysed post decision (73 DA; 73 control); 95 Prevention and 24 DCIS participants were analysed at follow-up (58 DA; 61 control). There was no effect on the primary outcome of decisional conflict. The DCIS-DA group had higher knowledge post decision, and the Prevention-DA group had lower decisional regret at follow-up.

Conclusions: This was the first study to evaluate a DA in the clinical trial setting. The results suggest DAs can potentially increase knowledge and reduce decisional regret about clinical trial participation.

Randomised controlled trials (RCTs) are regarded as the gold standard for evaluating medical interventions (Sackett et al, 1996), and research has demonstrated support for clinical trials as a means of improving medical care among oncology patients and the general public (Comis et al, 2003; Jenkins et al, 2010). However, patients commonly fail to understand the rationale and design of clinical trials, which compromises the process of informed consent (Fallowfield et al, 1998; Joffe et al, 2001a). Participants who do not fully understand what is involved in a trial before being randomised may be more likely to drop out, which can bias results and limit study conclusions (Wood et al, 2004). 
Informed consent requires that patients understand the information provided, and are able to reason about possible courses of action. However, information delivered to patients about clinical trials is often unclear, incomplete and inadequate (Jefford and Moore, 2008). Complex language and excessive detail in patient information statements and consent forms are likely to render these documents unreadable for many patients, and thus may confuse rather than enhance patient understanding (Sharp, 2004). Clinicians and data managers often report difficulty in explaining trials, and audio/video-tape audits show that critical information is often omitted or poorly presented during consent interviews (Jenkins et al, 1999; Loh et al, 2002). Only patients who understand their management options, including both standard care and clinical trial alternatives, can make informed choices about trial participation.

Decision aids (DAs) may be an effective way to optimise the informed consent process for clinical trials (Stacey et al, 2014). These decision-making tools supplement verbal guidance from clinicians by presenting clear written and graphical information about options and outcomes. They help patients to clarify their values and weigh up the pros and cons of their options before making a decision. A Cochrane review found that DAs improve patients' understanding and decision making in the context of other health decisions (i.e., not clinical trials), including increased knowledge of options and outcomes, more realistic expectations, less difficulty reaching a decision, more active participation in decision-making, no differences in anxiety levels and equivalent or greater decisional satisfaction, compared with controls (Stacey et al, 2014). A meta-analysis of cancer-specific screening and treatment contexts demonstrated improved knowledge and reduced decisional conflict among patients receiving DAs vs controls (O'Brien et al, 2009). Although there has been interest in using DAs to support decision making about clinical trial participation (Brehaut et al, 2009, 2010; Stepan et al, 2011), their efficacy has not been systematically evaluated in this context, and their influence on participant dropout is unknown. To our knowledge, this study is the first RCT of a DA specifically developed for a clinical trial.

The trial selected for this study was the International Breast Cancer Intervention Study-II (IBIS-II), an international multicentre study evaluating the role of anastrozole $v s$ standard treatment, in the management of women at increased risk of breast cancer (Cuzick, 2008). Two groups of post-menopausal women were recruited to IBIS-II as follows: (a) those at elevated risk of breast cancer without breast symptoms requiring treatment in the last 5 years (IBIS-II Prevention); and (b) those recently treated for ductal carcinoma in situ (DCIS), who were recruited from the traditional clinical setting (IBIS-II DCIS). Consequently, two variants of the DA could be used, allowing evaluation in both a prevention and clinical context.

Using a two-arm parallel group RCT design, the effect of provision of the DA $v s$ no DA was investigated in women potentially eligible to participate in the IBIS-II trial. Breast cancer chemoprevention was anticipated to be a suitable setting for a DA due to low uptake among high-risk women in spite of good evidence for its efficacy (Juraskova and Bonner, 2013). Furthermore, in the DA pilot (Juraskova et al, 2008), women indicated that the DA was very useful in helping them with this difficult decision. It was hypothesised that women receiving a DA prior to deciding whether to participate in IBIS-II would have lower decisional conflict and higher knowledge at the time of decision making, and report more satisfaction and less regret regarding their decision at 3-month follow-up, compared with women not receiving this intervention. The $\mathrm{DA}$ was also expected to reduce dropout due to improved informed consent, but not influence accrual, as it was developed as a non-directional, unbiased tool.

\section{MATERIALS AND METHODS}

The study was approved by the Human Research Ethics Committees of The University of Sydney and participating hospitals in Australia, New Zealand and the United Kingdom.

Decision aid. The development, detailed content and pilot testing of the DCISDA is reported elsewhere (Juraskova et al, 2007, 2008), and a similar process was used for the Prevention DA. The DAs included evidence-based representation of breast cancer risk, the IBIS-II trial rationale, explanation of management options available on and off the trial, a comparison of the risks and benefits of each option, and values clarification worksheets (full content available at: http://sydney.edu.au/science/psychology/ cemped/com_decision_aids.shtml). The DAs were designed to meet International Patient Decision Aid Standards (IPDAS) guidelines for content development, including sufficient detail about options, unbiased and understandable presentation of probabilities, values clarification exercises, structured guidance in deliberation, balanced presentation of options, up to date evidence with references, disclosed conflicts of interest and use of plain language (International Patient Decision Aid Standards Collaboration, 2005). Pilot data confirmed this with good understanding of the DA content ( $>80 \%$ correct on 12/13 items) and agreement that the DA was balanced (97\%), helpful for deciding about trial participation (97\%), and provided useful additional information to the trial information sheet (97\%) (Juraskova et al, 2008).

Participants. Women potentially eligible to participate in the IBIS-II trial were recruited to the DA study from 11 Australian, 1 New Zealand and 2 UK centres. These women were at elevated risk of breast cancer owing to family history (Prevention cohort) or recent treatment for DCIS (DCIS cohort). Women were excluded from the study if they had insufficient English to read the DA/questionnaires, were ineligible for IBIS-II $(n=10)$ or had already made a decision about the trial before reading the DA $(n=3)$. Women who had participated in the previous IBIS-I trial $(n=66)$ were excluded from the analyses because of their high pre-existing knowledge about trials, which was not anticipated a priori, as this would have created an artificial ceiling effect that did not reflect the usual decision-making process.

Procedure. For the Prevention cohort, clinical staff phoned potentially eligible women who had enquired about the IBIS-II trial (usually in response to media advertisements) and invited them to join the DA study using a standardised introduction. For the DCIS cohort, potentially eligible women were approached by their surgeon who introduced both the IBIS-II trial and DA study. Women who gave verbal consent to participate in the DA study and were still undecided regarding participation in IBIS-II were given a pre-randomised and sequentially numbered sealed package, with or without a DA. The package randomisation sequence was generated using the website www.randomizer.org. A 1:1 allocation ratio was used and packages were randomised in blocks of 10 according to centre to maintain balanced group sizes. Recruiters were blinded to group allocation, but research team members responsible for participant follow-up were not.

The DA group received the standard IBIS-II information sheet and consent form, the DA information sheet and consent form, a questionnaire and DA booklet. The control group received the same except a blank notebook replaced the DA to ensure recruiters remained blinded to randomisation. Both packages included a reply-paid envelope for return of the DA study consent form and questionnaire. A DA study researcher called participants 2 weeks after recruitment to confirm receipt of the package and remind 
women to return the DA study consent form and questionnaire. Three months after they returned the initial questionnaire, women were posted a second questionnaire and reply-paid envelope. Non-responders were followed up by phone and were re-sent questionnaires. Recruitment ceased when the IBIS-II trial was closed. Reasons for dropout are displayed in Figure 1.

Measures. The primary outcome for the study was difficulties with decision-making, which was assessed using the validated decisional conflict scale (DCS, O'Connor, 1995) after the trial participation decision had been made. The DCS contains 16 items designed to measure the amount of uncertainty a person has regarding a course of action, and factors contributing to that uncertainty. All items are answered on a 1-5 Likert scale, with responses ranging from 'strongly agree' to 'strongly disagree'. Summary scores are calculated by transforming the mean of all responses to a score out of 100 . Lower scores indicate less decisional conflict. The DCS has been found to have good internal consistency, with Cronbach's alpha coefficients ranging from 0.78 to 0.92 , and test-retest reliability coefficients $>0.80$ (O’Connor, 1995).

Several secondary outcomes were also assessed:

- Participants' knowledge about clinical trials generally and the IBIS-II trial specifically was assessed using the following scales:

(a) General knowledge about clinical trials, as presented in the DA, was assessed using an adapted form of an existing 7 -item scale used in the IBIS-II DCIS-DA pilot (Juraskova et al, 2007, 2008). (b) Objective understanding of IBIS-II was assessed using 12- and 16-item knowledge scales for the Prevention and DCIS cohorts respectively, developed specifically for this study.

(c) The 14-item Quality of Informed Consent scale - Part B was used to measure perceived/subjective understanding of IBIS-II. This scale has good test-retest reliability $(\mathrm{r}=0.77$, Joffe et al, 2001b), and our team has obtained psychometric data on this measure in the Australian context (Brown et al, 2004).

- Women's attitudes towards participating in IBIS-II were assessed using an adapted 9-item version of an existing attitude scale (Marteau et al, 2001).

- Anxiety levels were assessed using a 6-item short-form of the State-Trait Anxiety Inventory state scale. The short-form was selected to keep questionnaire length to a minimum. It produces similar scores to the full 20-item version, with acceptable reliability and validity, and sensitivity to fluctuations in state anxiety (Marteau and Bekker, 1992).

Demographics including age, marital status, education, occupation, medical training and medical conditions were also assessed.

The 3-month follow-up questionnaire assessed decisional satisfaction and regret regarding IBIS-II participation using the satisfaction with decision (SWD) scale and decisional regret scale (DRS), respectively. The 6-item SWD scale has good reliability and discriminant validity (Holmes-Rovner et al, 1996). The DRS is a

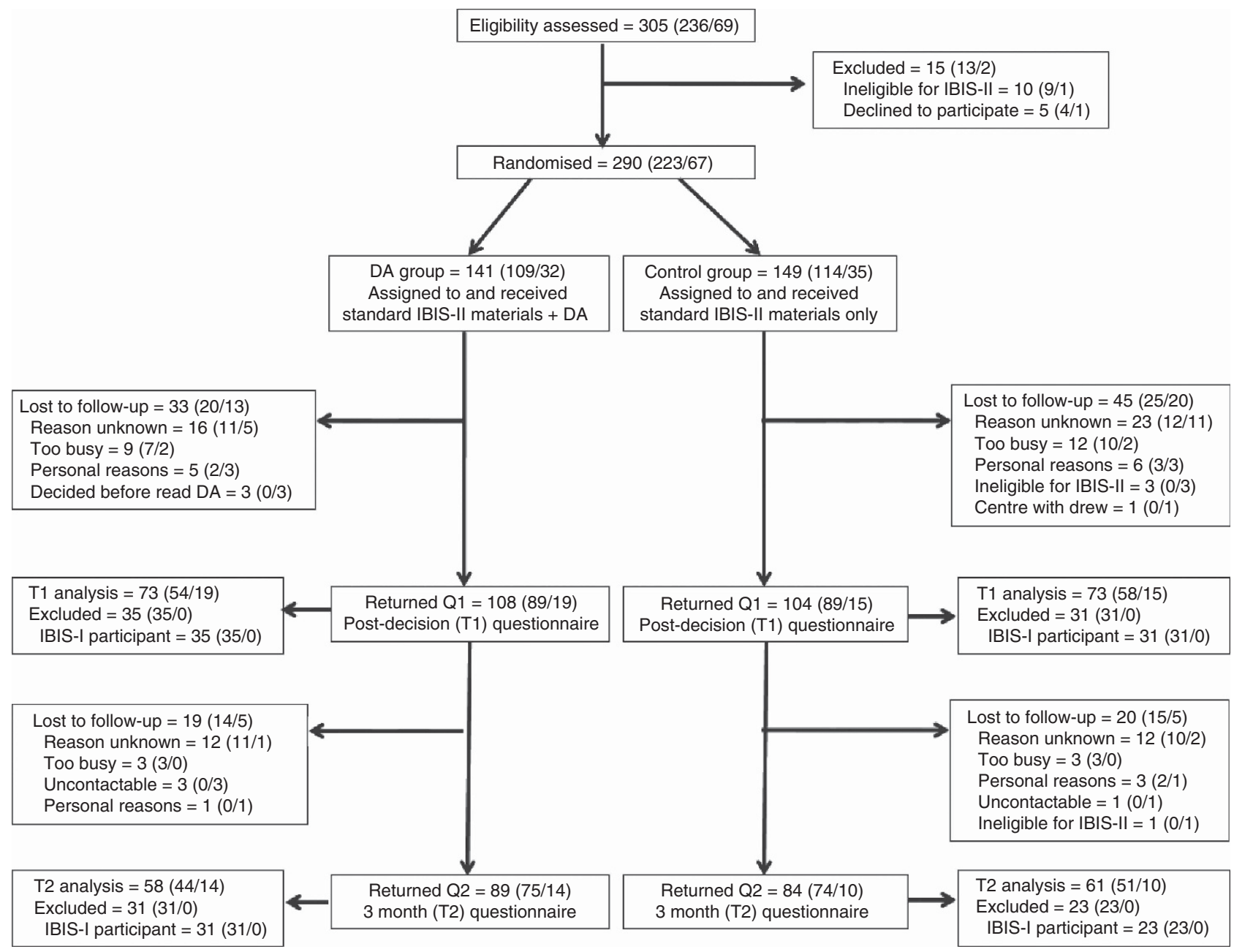

Figure 1. Recruitment flowchart: total (Prevention/DCIS). 
widely used 5-item scale with good internal consistency (Cronbach's alphas $=0.81-0.92)$ (Brehaut et al, 2003).

In terms of trial-related behaviour, intention to participate in IBIS-II was assessed at T1 after the trial participation decision had been made, and actual enrolment and subsequent dropout were recorded at the end of the trial.

Sample size and data analysis. A sample size of 64 participants per group (DA, control), per cohort (DCIS, Prevention) yields $80 \%$ power to detect a moderate (standardised) effect size of 0.5 in the primary outcome of decisional conflict or any of the secondary outcomes, assuming a two-sided alpha of 0.05 (Cohen, 1992).

Descriptive statistics were used to assess sample characteristics and perceptions of the DA. Unadjusted differences and 95\% confidence intervals (CI) in continuous outcomes between the DA and control groups, by cohort, were computed from a regression model including group, cohort and the interaction group by cohort variables. Adjusted models included age, education (high school or vocational training $v s$ university) and medical training (yes or no). Binary outcomes were assessed using logistic regression with the same variables as above. Although our primary interest was in the comparison of intervention group (i.e., DA vs control), we also were interested in whether the intervention effects differed by cohort, so the interaction terms were included and are reported. Standardised effect sizes were calculated by dividing the difference between the DA and control group by their pooled standard deviation (s.d.). Clinically important differences in mean values of patient reported outcomes have been estimated to be in the range of 0.3-0.5 (King, 2011).

\section{RESULTS}

The recruitment flowchart is provided in Figure 1. Across the Prevention and DCIS cohorts, 305 women were assessed for eligibility; 290 were randomised; 212 completed the first questionnaire post-decision (T1); 173 completed the second questionnaire at 3-month follow-up (T2); and 146 were included in the final analysis of the primary outcome.

The sample characteristics for each cohort are displayed in Table 1. Participants were on average 59-years-old and the majority were married, with high school or vocational education. All DA group participants except one DCIS participant looked through the DA, of whom most (86\%) read the DA 'quite thoroughly' or 'from cover to cover'. Most (81\%) agreed the DA made it easier to understand the IBIS-II trial than reading the IBIS-II Information sheet alone, and $86 \%$ agreed women should receive both the information sheet and the DA.

Primary outcome. There was no difference between the DA and control groups on the primary outcome of decisional conflict at T1 in either cohort. The Prevention cohort had very low levels of decisional conflict across both groups ( $M=15.7 \mathrm{DA} ; 13.2$ control), whereas the DA group in the DCIS cohort had a higher level ( $M=20.7$ DA; 11.9 control), but the difference between cohorts was not statistically significant.

Secondary outcomes: psychosocial. In the Prevention cohort, the DA group $(M=10.1)$ had lower decisional regret than the control group $(M=16.0)$ at $\mathrm{T} 2$ (difference $(95 \% \mathrm{CI})=-5.9$ $(-11.4,-0.4) ; P=0.04)$. Decisional satisfaction at this time point

Table 1. Sample characteristics by cohort and group

\begin{tabular}{|c|c|c|c|c|}
\hline \multirow[b]{2}{*}{ Characteristic } & \multicolumn{2}{|c|}{ Prevention cohort } & \multicolumn{2}{|c|}{ DCIS cohort } \\
\hline & $\mathrm{DA}(n=54)$ & Control $(n=58)$ & $\mathrm{DA}(n=19)$ & Control $(n=15)$ \\
\hline \multirow[t]{2}{*}{ Age (mean, s.d.) } & $59.2(5.9)$ & $59.2(5.3)$ & $58.5(3.9)$ & $58.7(5.0)$ \\
\hline & $n(\%)$ & $n(\%)$ & $n(\%)$ & $n(\%)$ \\
\hline Married & $43(80)$ & $47(81)$ & $15(79)$ & $7(47)$ \\
\hline \multicolumn{5}{|l|}{ Education } \\
\hline $\begin{array}{l}\text { High school/vocational } \\
\text { University or higher }\end{array}$ & $\begin{array}{l}40(75) \\
12(21)\end{array}$ & $\begin{array}{l}46(79) \\
13(25)\end{array}$ & $\begin{array}{r}13(72) \\
5(28)\end{array}$ & $\begin{array}{l}7(54) \\
6(46)\end{array}$ \\
\hline \multicolumn{5}{|l|}{ Occupation } \\
\hline $\begin{array}{l}\text { Managers/professionals } \\
\text { Other (e.g., trade, clerk, sales, driver) } \\
\text { Home duties }\end{array}$ & $\begin{array}{l}28(52) \\
22(41) \\
4(7)\end{array}$ & $\begin{array}{c}27(47) \\
27(47) \\
2(3)\end{array}$ & $\begin{array}{r}10(53) \\
7(37) \\
2(11)\end{array}$ & $\begin{array}{c}10(67) \\
3(20) \\
0(0)\end{array}$ \\
\hline \multicolumn{5}{|l|}{ Country of birth } \\
\hline $\begin{array}{l}\text { Australia } \\
\text { New Zealand } \\
\text { United Kingdom } \\
\text { Other }\end{array}$ & $\begin{aligned} 31(57) \\
12(22) \\
9(17) \\
2(4)\end{aligned}$ & $\begin{array}{l}40(69) \\
10(17) \\
5(9) \\
3(5)\end{array}$ & $\begin{array}{l}0(0) \\
4(21) \\
9(47) \\
0(0)\end{array}$ & $\begin{array}{c}0(0) \\
2(13) \\
11(73) \\
0(0)\end{array}$ \\
\hline \multicolumn{5}{|l|}{ Language spoken } \\
\hline $\begin{array}{l}\text { English only } \\
\text { Other languages }\end{array}$ & $\begin{array}{c}53(98) \\
1(2)\end{array}$ & $\begin{array}{l}57(98) \\
1(2)\end{array}$ & $\begin{array}{r}17(89) \\
2(11)\end{array}$ & $\begin{array}{c}14(93) \\
1(7)\end{array}$ \\
\hline Medical/allied health training & $12(23)$ & $16(28)$ & $3(16)$ & $3(20)$ \\
\hline Chronic medical condition & $15(28)$ & $16(28)$ & $2(11)$ & $4(27)$ \\
\hline
\end{tabular}


was higher in the DA group $(M=4.62)$ than the control group $(M=4.42)$ but this did not reach statistical significance (difference $(\mathrm{CI})=0.20(-0.42,0.02) ; P=0.07)$. There were no significant differences between groups on these outcomes in the DCIS cohort.

In the DCIS cohort, the DA group $(M=77.6)$ had higher objective knowledge about IBIS-II than the control group $(M=63.8)$ at $\mathrm{T} 1$ (difference $(\mathrm{CI})=13.9(3.7,24.0) ; P=0.008)$. Anxiety at this time point was lower in the DA group $(M=29.6)$ than the control group $(M=36.7)$ but this did not reach statistical significance (difference $(\mathrm{CI})=-7.1(-14.7,0.6) ; P=0.07)$. There were no significant differences between groups on these outcomes in the Prevention cohort.

There were no significant differences between the DA and control groups for any other psychosocial outcomes in either cohort, and the adjusted means were similar (see Tables 2 and 3). There were statistically significant cohort effects for general clinical trials knowledge, knowledge about IBIS-II (objective and subjective), intention to participate and dropout. These were all higher for the Prevention cohort, except for dropout, which was lower. There were statistically significant cohort by group interactions, indicating a difference in the intervention effect between cohorts, for objective knowledge of IBIS-II and for anxiety. The latter was driven by high values in the DCIS-control group. See Tables 2 and 3.

Secondary outcomes: trial-related behaviour. There was no significant effect of the DA on intention to participate at $\mathrm{T} 1$, actual enrolment in the IBIS-II trial, or subsequent dropout. A larger proportion of enrolled participants dropped out from the DCIS cohort (36\% DA; $22 \%$ control) compared with the Prevention cohort (12\% DA; $8 \%$ control), but the numbers involved were small. See Tables 2 and 3.

\section{DISCUSSION}

This study investigated the effect of decision aids (DAs) in a new context: a clinical trial for women at increased risk of breast cancer, in both prevention and clinical (DCIS) settings. The DAs did not have an effect on the primary outcome, decisional conflict. The standard of information and support provided by IBIS-II clinicians and trial coordinators may have been high enough without the DA to see any effect on decisional conflict. For instance, investigator meetings for the trial included communication skills training with an actor, which many investigators participated in. The efficacy of the DAs could perhaps have been improved if clinicians were instructed to go through the DA with the women, including their responses to the values clarification worksheets.

There is no consensus on the best way to measure the quality of decision making, so decisional conflict was chosen as the primary outcome because it is the most commonly used measure in DA studies (Sepucha et al, 2013). However, a recent review by the IPDAS collaboration highlights the importance of both the decision-making process (e.g., feeling informed about options and outcomes, feeling clear about goals and preferences) and decision quality (e.g., knowledge, realistic expectations) (Sepucha et al, 2013). Our significant findings for decisional regret and knowledge are therefore important despite being originally defined as secondary outcomes, and arguably relate more to decision quality than the decision-making process. The results suggest that DAs may improve the informed consent process by increasing knowledge and reducing decisional regret. However, different results were found in the Prevention and DCIS cohorts, suggesting that trial population characteristics are important in determining intervention efficacy.

The DA improved knowledge in the clinical (DCIS) setting but not in the prevention setting. The prevention group were volunteers who approached cancer centres in response to media advertisements, giving them more time to research the trial prior to participation in the DA study. They had higher scores than the DCIS cohort on all knowledge measures in both DA and control groups, leaving little room for improvement as a result of the DA. In contrast, the DCIS-DA groups had improved knowledge after reading the DA relative to the control group, indicating that DAs can improve informed consent relative to standard consent materials in the clinical setting, where patients were making the decision after treatment and had more hormonal treatment options to consider (Anastrozole vs Tamoxifen).

Three months after deciding about trial participation, the DA group had lower decisional regret, although this effect was higher in the prevention setting (standardised effect -0.44 ) than the clinical setting (standardised effect -0.24 ). Decisional regret is theoretically important to measure because patients may continue to consolidate or 'react to and interpret' their decision after the decision has been made (Feldman-Stewart et al, 2012; Sepucha et al, 2013).

Table 2. Primary and secondary outcomes for the Prevention cohort (Unadjusted results shown)

\begin{tabular}{|c|c|c|c|c|c|}
\hline Outcome & DA mean (s.d.) & Control mean (s.d.) & Difference $(95 \% \mathrm{Cl})$ & $P$-value & Standardised effect \\
\hline Decisional conflict (DCS; $\max 100)$ & $15.7(14.7)$ & $13.2(14.5)$ & $2.6(-8.4,3.3)$ & 0.4 & 0.18 \\
\hline Knowledge of clinical trials $(\max 7)$ & $5.88(1.2)$ & $5.91(1.4)$ & $-0.03(0.53,-0.47)^{a}$ & 0.9 & -0.02 \\
\hline Objective knowledge of IBIS-II (max 100) & $81.1(14.0)$ & $80.4(12.7)$ & $-0.08(-0.85,0.68)^{a, b}$ & 0.8 & 0.05 \\
\hline Subjective knowledge of IBIS-II (QulC-B; $\max 100)$ & $93.4(10.1)$ & $92.0(9.9)$ & $1.5(-6.3,3.6)^{a}$ & 0.6 & 0.15 \\
\hline Anxiety (STAI-S; $\max 80$ ) & $31.0(10.7)$ & $29.6(10.0)$ & $1.4(-6.6,3.3)^{\mathbf{b}}$ & 0.5 & 0.14 \\
\hline Attitude towards IBIS-II (max 100) & $83.6(13.9)$ & $83.9(14.7)$ & $-0.31(-5.3,5.9)$ & 0.9 & -0.02 \\
\hline Decisional regret at 3 months (DRS; $\max 50$ ) & $10.1(11.8)$ & $16.0(14.1)$ & $-5.9(-11.4,-0.4)$ & 0.04 & -0.44 \\
\hline Decisional satisfaction at 3 months (SWD; $\max 5$ ) & $4.62(0.4)$ & $4.42(0.5)$ & $0.20(-0.42,0.02)$ & 0.07 & 0.41 \\
\hline Intended to participate in IBIS-II & $65 \%$ & $64 \%$ & $2(-16,19)$ & 0.9 & 0.02 \\
\hline Actually enrolled in IBIS-II & $50 \%$ & $43 \%$ & $7(-12,26)$ & 0.5 & 0.14 \\
\hline Dropped out from IBIS-II ${ }^{\mathrm{c}}$ & $3 / 26=12 \%$ & $2 / 25=8 \%$ & $4(-13,20)^{b}$ & 0.7 & 0.12 \\
\hline \multicolumn{6}{|c|}{$\begin{array}{l}\text { Abbreviations: } \mathrm{Cl}=\text { confidence interval; DCS = decisional conflict scale; DRS = Decisional Regret Scale; IBIS-II = Internation } \\
\text { Informed Consent scale }- \text { Part } B ; \text { STAI-S = State-Trait Anxiety Inventory state scale; SWD = Satisfaction with Decision scale. } \\
\mathrm{a}_{P}<0.05 \text { for main effect of cohort, indicating a difference in levels between Prevention and DCIS cohorts. } \\
\mathrm{b}_{P}<0.05 \text { for main effect of cohort } x \text { group, indicating a difference in intervention effect between Prevention and DCIS cohorts. } \\
{ }^{c} \text { Among those who enrolled. }\end{array}$} \\
\hline
\end{tabular}


Table 3. Primary and secondary outcomes for the DCIS cohort (Unadjusted results shown)

\begin{tabular}{|c|c|c|c|c|c|}
\hline Outcome & DA mean (s.d.) & Control mean (s.d.) & Difference $(95 \% \mathrm{Cl})$ & $P$-value & Standardised effect \\
\hline Decisional conflict (DCS; max 100) & $20.7(25.4)$ & $11.9(10.9)$ & $8.8(-2.4,20.0)$ & 0.1 & 0.42 \\
\hline Knowledge of clinical trials $(\max 7)$ & $5.56(1.4)$ & $5.14(1.8)$ & $0.41(-0.52,1.35)^{a}$ & 0.3 & 0.26 \\
\hline Objective knowledge of IBIS-II (max 100) & $77.6(16.5)$ & $63.8(22.2)$ & $13.9(3.7,24.0)^{a, b}$ & 0.008 & 0.69 \\
\hline Subjective knowledge of IBIS-II (QuIC-B; max 100) & $88.9(12.5)$ & $83.2(25.6)$ & $5.7(-3.3,14.6)^{a}$ & 0.2 & 0.29 \\
\hline Anxiety (STAI-S; $\max 80$ ) & $29.6(8.7)$ & $36.7(10.9)$ & $-7.1(-14.7,0.6)^{b}$ & 0.07 & -0.70 \\
\hline Attitude towards IBIS-II (max 100) & $76.0(15.3)$ & $83.2(11.8)$ & $-7.1(-17.2,2.9)$ & 0.2 & -0.51 \\
\hline Decisional regret at 3 months (DRS; $\max 50$ ) & $16.9(17.4)$ & $20.5(12.1)$ & $-3.5(-14.6,7.4)$ & 0.5 & -0.24 \\
\hline Decisional satisfaction at 3 months (SWD; $\max 5$ ) & $4.40(0.7)$ & $4.39(0.4)$ & $0.01(-0.41,0.41)$ & 1.0 & 0.01 \\
\hline Intended to participate in IBIS-II & $65 \%$ & $73 \%$ & $-9(-41,23)$ & 0.6 & -0.09 \\
\hline Actual enrolment in IBIS-II & $58 \%$ & $64 \%$ & $-6(-40,27)$ & 0.7 & -0.13 \\
\hline Dropout from IBIS-II ${ }^{c}$ & $4 / 11=36 \%$ & $2 / 9=22 \%$ & $14(-25,53)^{\mathbf{b}}$ & 0.4 & 0.30 \\
\hline \multicolumn{6}{|c|}{ 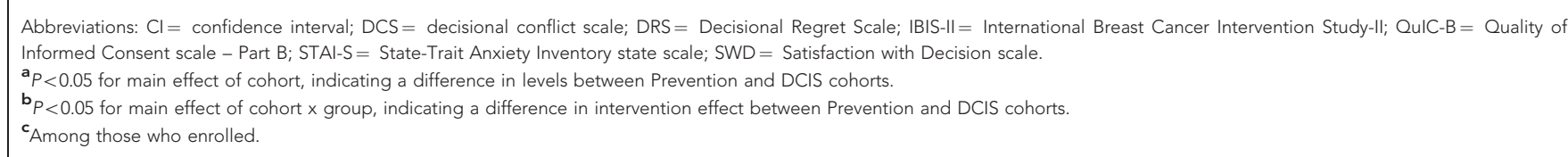 } \\
\hline
\end{tabular}

Therefore, the decision-making process does not necessarily end once an initial decision has been made. This is an interesting finding as the most recent review of DA efficacy indicates that previous DA studies have not found any effect on decisional regret (Stacey et al, 2014). In the context of our study, the decisional regret effect could be interpreted as the DA participants having more realistic expectations because they had explicitly considered the possible downsides of participating, including how they would feel about side effects and the inconvenience of taking a pill every day. Future research on clinical trial DAs could include measures of the potential downsides of trial participation, such as side effects, to identify the reasons for any effect on decisional regret, including long-term outcomes.

The main limitation of this study is that it was underpowered, particularly for the clinical DCIS cohort, where standardised effect sizes of 0.4 and greater were not found to be statistically significant, despite these being in the moderate effect size range (Cohen, 1992; King, 2011). The Australia/New Zealand centres had more success recruiting participants for the Prevention arm of the IBIS-II trial than the DCIS arm (657 vs 130), and not all centres could participate in the DA study. An attempt was made to address this issue by involving centres from the UK at a later stage. This improved recruitment but the UK data collection period was limited owing to the closure of the IBIS-II trial. The analyses involved testing of multiple outcomes, and additional insights may have been gained by including long-term outcomes. The need to exclude IBIS-I participants owing to their high pre-existing knowledge about trials was not anticipated in advance. Future research should consider prior trial participation a priori. There was also additional dropout, but the rates and reasons provided were similar in the two arms.

Further investigation is needed to identify the trial population characteristics that result in the most benefit from DAs. This study found that a clinical setting benefited more than a volunteer prevention setting in terms of knowledge, but the decisional conflict and regret results are less clear. A DA for the RadiotherapyAdjuvant vs Early Salvage prostate cancer clinical trial has been piloted and is currently being evaluated in a national RCT, which may provide further understanding of these variables (Sundaresan et al, 2011). The current study suggests some methodological improvements that could be made in future DA studies within clinical trials: involve all trial centres from the start of recruitment as part of the clinical trial itself; select a trial population likely to have lower levels of knowledge and more complex treatment options; and measure decisional conflict at a consistent time point, as close as possible to actual trial enrolment. Comparison with other types of interventions to improve informed consent, such as question prompt lists, would also be useful (Kinnersley et al, 2013).

\section{CONCLUSION}

This was the first RCT of a DA in the clinical trial setting. Although there was no effect on the primary outcome of decisional conflict, the results suggest that DAs can potentially increase knowledge and reduce decisional regret about clinical trial participation. Further research is needed to identify the underlying mechanisms for these effects, and the ideal trial populations for which DAs are beneficial.

\section{ACKNOWLEDGEMENTS}

We thank all the participants, staff at the Australia and New Zealand Breast Cancer Trials Group (ANZBCTG) operations centre in Newcastle, Australia, and the principal investigators and trial coordinators at Armidale Base Hospital (Nick Pavlakis, Pamela Witten), Coffs Harbour Health Campus (Karen Briscoe, Joanne Smith), Newcastle Calvary Mater Hospital (John Forbes, Vicki Sproule), Royal Melbourne Hospital (A/Prof John Collins, Gillian Hoogeveen), Sir Charles Gairdner Hospital (Christobel Saunders, Suzanne Wegecsanyi), St Vincent's Hospital Melbourne (Raymond Snyder, Sarah Vickery), St Vincent's Hospital Sydney (Robyn Ward, Lynne Jolly), Tamworth Hospital (Elizabeth Hovey, Rebecca Hemmings, Margaret Chamen), The Breast Centre (David Clark, Judith Silcock), Waikato Hospital (Ian Campbell, Shelley Cavanagh), Liverpool Hospital (Chris Holcombe, Laura Francis), and Southampton Hospital (Ramsey Cutress, Kim Stevens, Julie Gwilt). We thank Joseph Coll and Daniel Costa for assistance with analyses.This study was supported by Susan G. Komen for the Cure (grant number BCTR0503961) and discretionary funding from the Breast Cancer Institute of Australia, which is the fundraising and education department of the Australia and New Zealand Breast Cancer 
Trials Group (ANZBCTG; no grant number). The funding bodies had no role in the writing of the manuscript or decision to submit it for publication. The authors were not paid by a pharmaceutical company or other agency to write this article. Dr Ilona Juraskova had full access to all the data in the study and had final responsibility for the decision to submit for publication. Australian New Zealand Clinical Trials Registry (ANZCTR) numbers are as follows: IBIS-II DA study: ACTRN126050005 06695, IBIS-II Prevention: ACTRN12605000216617 and IBIS-II DCIS: ACTRN12605000282684.

\section{CONFLICT OF INTEREST}

The authors declare no conflict of interest.

\section{AUTHORS CONTRIBUTION}

Ilona Juraskova-conception, collection, analysis, interpretation, writing; Phyllis Butow-conception, collection, analysis, interpretation; Carissa Bonner-collection, analysis, interpretation, writing; Melanie L Bell-analysis, interpretation, writing; Allan B Smithcollection, analysis, interpretation; Margaret Seccombeconception, collection; Fran Boyle-recruitment, interpretation; Linda Reaby (consumer) - collection, interpretation; Jack Cuzickanalysis, interpretation; John F Forbes-conception, collection, interpretation

\section{REFERENCES}

Brehaut JC, O'Connor AM, Wood TJ, Hack TF, Siminoff L, Gordon E, Feldman-Stewart D (2003) Validation of a decision regret scale. Med Decis Making 23: 281-292.

Brehaut JC, Saginur R, Elwyn G (2009) Informed consent documentation necessary but not sufficient. Contemp Clin Trials 30: 388-389.

Brehaut JC, Fergusson DA, Kimmelman J, Shojania K, Saginur R, Elwyn G (2010) Using decision aids may improve informed consent for research. Contemp Clin Trials 31: 218-220.

Brown RF, Butow PN, Ellis P, Boyle F, Tattersall MHN (2004) Seeking informed consent to cancer clinical trials: describing current practice. Soc Sci Med 58: 2445-2457.

Cohen J (1992) A Power Primer. Psychol Bull 112: 155-159.

Comis RL, Miller JD, Aldigé CR, Krebs L, Stoval E (2003) Public attitudes toward participation in cancer clinical trials. J Clin Oncol 21: $830-835$.

Cuzick J (2008) IBIS II: a breast cancer prevention trial in postmenopausal women using the aromatase inhibitor anastrozole. Expert Rev Anticancer Ther 8: 1377-1385.

Fallowfield LJ, Jenkins V, Brennan C, Sawtell M, Moynihan C, Souhami RL (1998) Attitudes of patients to randomised clinical trials of cancer therapy. Eur J Cancer 34: 1554-1559.

Feldman-Stewart D, Tong C, Siemens R, Alibhai S, Pickles T, Robinson J, Brundage MD (2012) The impact of explicit values clarification exercises in a patient decision aid emerges after the decision is actually made: evidence from a randomized controlled trial. Med Decis Making 32: 616-626.

Holmes-Rovner M, Kroll J, Schmitt N, Rovner DR, Breer ML, Rothert ML, Padonu G, Talarczyk G (1996) Patient satisfaction with health care decisions: the satisfaction with decision scale. Med Decis Making 16: 58-64.

International Patient Decision Aid Standards Collaboration (2005) Criteria for Judging the Quality of Patient Decision Aid Standards. Available at http://www.ipdas.ohri.ca/.

Jefford M, Moore R (2008) Improvement of informed consent and the quality of consent documents. Lancet Oncol 9: 485-493.

Jenkins VA, Fallowfield LJ, Souhami A, Sawtell M (1999) How do doctors explain randomised clinical trials to their patients? Eur J Cancer 35: 1187-1193.
Jenkins V, Farewell D, Batt L, Maughan T, Branston L, Langridge C, Parlour L, Farewell V, Fallowfield L (2010) The attitudes of 1066 patients with cancer towards participation in randomised clinical trials. Br J Cancer 103: 1801-1807.

Joffe S, Cook EF, Cleary PD, Clark JW, Weeks JC (2001a) Quality of informed consent in cancer clinical trials: a cross-sectional survey. Lancet 358: 1772-1777.

Joffe S, Cook EF, Cleary PD, Clark JW, Weeks JC (2001b) Quality of Informed Consent: a New Measure of Understanding Among Research Subjects. J Natl Cancer Inst 93: 139-147.

Juraskova I, Bonner C (2013) Decision aids for breast cancer chemoprevention. Breast Cancer Res 15: 106.

Juraskova I, Butow P, Lopez AL, Seccombe M, Boyle F, McCarthy N, Forbes JF (2007) Improving informed consent in clinical trials: Successful piloting of a decision aid. J Clin Oncol 25: 1443-1444.

Juraskova I, Butow P, Lopez A, Seccombe M, Coates A, Boyle F, McCarthy N, Reaby L, Forbes JF (2008) Improving informed consent: pilot of a decision aid for women invited to participate in a breast cancer prevention trial (IBIS-II DCIS). Health Expectations 11: 252-262.

King MT (2011) A point of minimal important difference (MID): a critique of terminology and methods. Expert Rev Pharmacoecon Outcomes Res 11: 171-184.

Kinnersley P, Phillips K, Savage K, Kelly MJ, Farrell E, Morgan B, Whistance R, Lewis V, Mann MK, Stephens BL, Blazeby J, Elwyn G, Edwards AG (2013) Interventions to promote informed consent for patients undergoing surgical and other invasive healthcare procedures. Cochrane Database Syst Rev 7: doi:10.1002/14651858.CD009445.pub2.

Loh WY, Butow PN, Brown RF, Boyle F (2002) Ethical communication in clinical trials - Issues faced by data managers in obtaining informed consent. Cancer 95: 2414-2421.

Marteau TM, Bekker H (1992) The development of a six-item short-form of the state scale of the Spielberger State-Trait Anxiety Inventory (STAI). Br J Clin Psychol 31: 301-306.

Marteau TM, Dormandy E, Michie S (2001) A measure of informed choice. Health Expect 4: 99-108.

O'Brien MA, Whelan TJ, Villasis-Keever M, Gafni A, Charles C, Roberts R, Schiff S, Cai W (2009) Are cancer-related decision aids effective? A systematic review and meta-analysis. J Clin OncolO 27: 974-985.

O'Connor A (1995) Validation of a decisional conflict scale. Med Decis Making 15: 25-30.

Sackett DL, Rosenberg WM, Gray JA, Haynes RB, Richardson WS (1996) Evidence based medicine: What it is and what it isn't-It's about integrating individual clinical expertise and the best external evidence. $\mathrm{Br}$ Med J 312: 71-72.

Sepucha KR, Borkhoff CM, Lally J, Levin CA, Matlock DD, Ng CJ, Ropka ME, Stacey D, Joseph-Williams N, Wills CE, Thomson R (2013) Establishing the effectiveness of patient decision aids: key constructs and measurement instruments. BMC Med Inform Decis Mak 13: S12.

Sharp M (2004) Consent documents for oncology trials: does anybody read these things? Am J Clin Oncol 27: 570-575.

Stacey D, Légaré F, Col NF, Bennett CL, Barry MJ, Eden KB, Holmes-Rovner M, Llewellyn-Thomas H, Lyddiatt A, Thomson R, Trevena L, Wu JH (2014) Decision aids for people facing health treatment or screening decisions. Cochrane Database Syst Rev 1: CD001431.

Stepan KA, Gonzalez AP, Dorsey VS, Frye DK, Pyle ND, Smith RF, Throckmorton TA, Villejo LA, Cantor SB (2011) Recommendations for enhancing clinical trials education: a review of the literature. J Cancer Educ 26: $64-71$.

Sundaresan P, Turner S, Kneebone A, Pearse M, Butow P (2011) Evaluating the utility of a patient decision aid for potential participants of a prostate cancer trial (RAVES-TROG 08.03). Radiother Oncol 101: 521-524.

Wood AM, White IR, Thompson SG (2004) Are missing outcome data adequately handled? A review of published randomized controlled trials in major medical journals. Clin Trials 1: 368.

This work is published under the standard license to publish agreement. After 12 months the work will become freely available and the license terms will switch to a Creative Commons AttributionNonCommercial-Share Alike 3.0 Unported License. 